\title{
Structural and magnetic studies in ferrihydrite nanoparticles formed within organic-inorganic hybrid matrices
}

\author{
N. J. O. Silva, ${ }^{\text {a) }}$ V. S. Amaral, and L. D. Carlos \\ Departamento de Física e CICECO, Universidade de Aveiro, Campus de Santiago, \\ P-3810-193 Aveiro, Portugal \\ B. Rodríguez-González and L. M. Liz-Marzán \\ Departmento de Quimica Fisica, Universidade de Vigo, 36310 Vigo, Spain
}

\begin{abstract}
A. Millan and F. Palacio
Instituto de Ciência de Materiales de Aragón, Universidad de Zaragoza-CSIC, 50009 Zaragoza, Spain

V. de Zea Bermudez

Departmento de Química and CQ-VR, Universidade de Trás-os-Montes e Alto Douro, Quinta de Prados, Apartado 1013, 5001-801 Vila Real, Portugal
\end{abstract}

(Received 10 March 2006; accepted 10 June 2006; published online 1 September 2006)

\begin{abstract}
We report detailed transmission electron microscopy, high resolution transmission electron microscopy (HRTEM), and scanning transmission electron microscopy/energy dispersive x-ray spectroscopy (STEM/EDS) studies on ferrihydrite nanoparticles in an organic-inorganic matrix. The Fourier transform of HRTEM images indicates the existence of six-line ferrihydrite. Combined STEM and EDS studies give further confirmation of the presence of iron in the observed particles and its absence in the matrix. The derived mean particle size and size distribution is $4.7 \pm 0.2 \mathrm{~nm}$ with a lognormal deviation of $s=0.4 \pm 0.1$. These values were used for analysis of magnetic measurements, yielding the determination of the anisotropy constant $K_{\text {eff }}=4 \times 10^{5} \mathrm{erg} / \mathrm{cm}^{3}$ and the power relation between the number of iron ions per particle and the number of uncompensated ones $p \approx 1 / 3$. This value indicates that the uncompensated spins are mainly randomly distributed at the surface. According to this model, a shell thickness of about one ferrihydrite unit cell is estimated.

(C) 2006 American Institute of Physics. [DOI: 10.1063/1.2336083]
\end{abstract}

\section{INTRODUCTION}

The term ferrihydrite applies to a type of nanocrystalline iron hydroxides that typically forms after rapid hydrolysis of iron salts at low $p \mathrm{H}$ and low temperatures. The importance of ferrihydrite in the environmental iron cycle and in the metallurgy process triggered the interest of the scientific community. ${ }^{1}$ Ferrihydrite is present in cold-water springs, recent bottom sediments of some lakes and soils as Hawaii basalt crusts, spodosols, and loesses. ${ }^{1}$ Ferrihydrite is also part of the iron cycle in living organisms. In fact, ferritin is a protein where $\mathrm{Fe}(\mathrm{III})$ is stored as ferrihydrite. ${ }^{2}$ The ferritin core holds up to 4000 iron atoms in a $5 \mathrm{~nm}$ radius ferrihydrite particle, ${ }^{2}$ exhibiting an interesting magnetic behavior due to the surface canted spins and their coupling with the inner spins. The magnetic properties of ferritin were intensely studied due to the possible existence of macroscopic quantum tunneling fluctuations. ${ }^{3}$ In antiferromagnetic particles as ferrihydrite, the dependence of magnetic moment with volume is not straightforward, since it arises from uncompensated/canted spins, and their number depends on size and on disorder. ${ }^{4}$ Therefore, comparing size distribution with moment distribution can give important information about the location of the uncompensated spins and about disorder.

In general, two kinds of ferrihydrite can be distin-

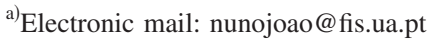

guished, according to the standard terminology: two-line ferrihydrite (2lFh) and six-line ferrihydrite (6lFh). ${ }^{1,5}$ The standard x-ray diffraction pattern of ferrihydrite (61Fh) shows peaks at $0.15,0.17,0.20,0.22-0.23$, and $0.25 \mathrm{~nm}$, while that for ferrihydrite (2lFh) shows basically two broad peaks at 0.15 and $0.25-0.26 \mathrm{~nm} .{ }^{1}$ The origin of these differences in the diffractograms seems not to be sufficiently understood, and therefore various models have been proposed to justify them. Drits et al. suggested that the differences between both kinds of ferrihydrites stem from a different coherence length due to the size of the crystalline domains. ${ }^{6}$ Schwertmann et al. have studied the evolution from iron nitrite salt to $61 \mathrm{Fh}$, and $2 \mathrm{lFh}$ does not seem to be an intermediate product but formed via a different route. ${ }^{7}$

Ferrihydrite obtained from aqueous media consists of agglomerated/coalesced isometric particles with typical domain size within a range between 3 and $10 \mathrm{~nm}$ determined using electron microscopy. ${ }^{8}$ Concerning applications and the systematic study of physical properties it is desirable to have nanoparticles with controlled size, shape, and interparticle distance. Among other methods, the formation of magnetic nanoparticles has been approached through the use of biological or other templates. Examples of the former include the use of ferritin ${ }^{3}$ and viral protein cages, ${ }^{9}$ zeolites, ${ }^{10}$ and polymers. ${ }^{11}$ We have recently reported the synthesis of ferrihydrite nanoparticles in a hybrid organic-inorganic matrix. ${ }^{12}$ The matrix, named diureasil, consists of a siliceous backbone covalently grafted to poly(oxyethylene) (POE) chains of two 
distinct average molecular weights $M_{w}$ (approximately 40.5 and 15.5 repeating units) by means of urea $[\mathrm{NHC}(=\mathrm{O}) \mathrm{NH}]$ groups. The formation of particles depends on the polymer length and benefits from the existence of carbonyl groups at the organic-inorganic interface. The mean particle size (diameter) can vary from 4 to $10 \mathrm{~nm}$ depending on the iron concentration (in the range of $1-6 \mathrm{wt} \%) .{ }^{13}$ Previous works were devoted to study the evolution of some structural ${ }^{13}$ and magnetic ${ }^{14}$ properties with the iron concentration. In this work we present a detailed transmission electron microscopy (TEM), scanning transmission electron microscopy/energy dispersive x-ray spectroscopy (STEM/EDS), and magnetic property studies of the ferrihydrite/organic-inorganic hybrid sample with $2.1 \%$ of iron. This detailed study allows a better understanding of the magnetic properties related to size, such as the blocking temperature and the magnetic moment.

\section{EXPERIMENT}

\section{Sample preparation}

The synthesis of iron-doped diureasils has been described in detail elsewhere. ${ }^{14}$ The preliminary step of the diureasil preparation involved the formation of urea linkages between the terminal $\mathrm{NH}$ groups of a doubly functional amine $[\alpha, \omega$-diamine poly(oxyethylene-co-oxypropylene) $]$ commercially available as Jeffamine ED-2001® (Fluka, $\left.M_{w}=2000\right)$ and the isocyanate group of an alkoxysilane precursor (3-isocyanatepropyltriethoxysilane, ICPTES, Fluka) in tetrahydrofuran (THF) (Merck) at room temperature (RT). ${ }^{15}$ A cross-linked hybrid precursor was, thus, obtained. The iron(III) nitrate nonahydrate $\left[\mathrm{Fe}\left(\mathrm{NO}_{3}\right)_{3} \cdot 9 \mathrm{H}_{2} \mathrm{O}\right.$, Aldrich] was incorporated in the second stage of the synthetic procedure. An appropriate amount of this salt was dissolved in a mixture of ethanol $\left(\mathrm{CH}_{3} \mathrm{CH}_{2} \mathrm{OH}\right)$ and water (molar ratio, ICPTES $\left./ \mathrm{CH}_{3} \mathrm{CH}_{2} \mathrm{OH} / \mathrm{H}_{2} \mathrm{O}=1: 4: 1.5\right)$. This solution was added to the nonhydrolyzed hybrid precursor prepared in the first stage. The resulting mixture was then stirred in a sealed flask for a few minutes at RT. The solution was cast into a mold. Gelation took place immediately. The mold was then transferred to an oven at ca. $40{ }^{\circ} \mathrm{C}$ for a period of seven days. The sample was obtained after aging for three weeks at ca. $80{ }^{\circ} \mathrm{C}$. The latter thermal treatment is crucial to yield mechanically stable films and to precipitate the ferrihydrite nanoparticles. The sample on which attention was focused in the present report was termed dU61Fh.

\section{Powder x-ray diffraction (XRD)}

Measurements were performed at RT with a Philips X'Pert MPD diffractometer using monochromated $\mathrm{Cu} \mathrm{K} \alpha$ radiation $(\lambda=1.541 \AA)$ in the $1.5^{\circ}-70^{\circ} 2 \theta$ range at $0.05^{\circ}$ resolution and $35 \mathrm{~s}$ acquisition per step.

\section{TEM}

Samples were prepared by grinding a small piece of the hybrid with a mortar, in the presence of $n$-butanol, then sonicating the resulting dispersion in a glass vial for $10 \mathrm{~min}$, and finally depositing and drying a droplet with a pipette on a copper grid covered with a carbon-coated, holey Formvar

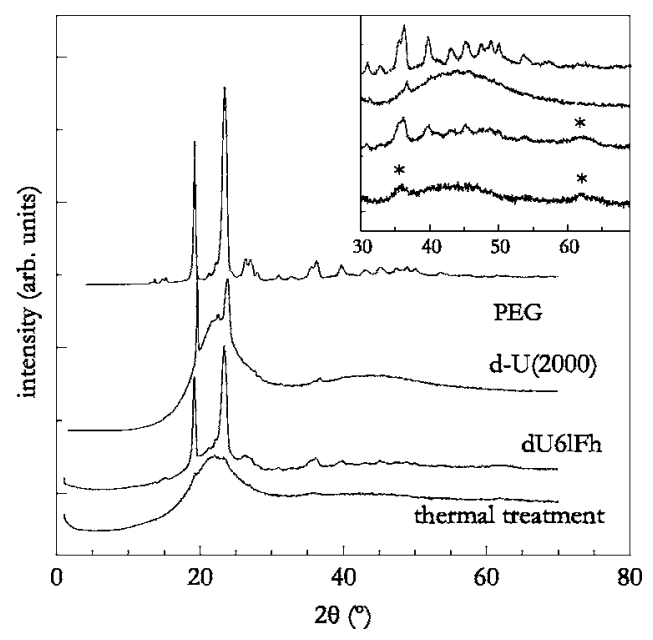

FIG. 1. XRD patterns of poly(oxyethyleneglicol) (PEG), the d-U(2000) matrix, the ferrihydrite/diureasil sample (dU61Fh), and the ferrihydrite/ diureasil sample after a thermal treatment performed to highlight the presence of ferrihydrite. Inset: zoom over the ferrihydrite features region.

polymer film. Microanalysis and imaging were performed on sample fragments located on holes, so that the electron beam is transmitted through the sample only. Images were obtained using a JEOL JEM 2010 FEG transmission electron microscope, operating at an acceleration voltage of $200 \mathrm{kV}$, equipped with a slow scan digital camera, a STEM unit, and an Oxford Inca Energy 200 energy EDS. Image Fourier filtrations were obtained from high resolution transmission electron microscopy (HRTEM) images by windowing the Fourier transform (FT). STEM images were recorded using a bright field detector, while for elemental mapping the STEM unit was coupled to the microanalysis system. Image analysis was carried out with DIGITAL MICROGRAPH software by Ga$\tan$.

\section{Magnetic measurements}

Magnetization data as a function of temperature and field were collected on a superconducting quantum interference device (SQUID) magnetometer (model MPMS2, Quantum Design Inc.) at IFIMUP, Universidade do Porto. The magnetic ac susceptibility was recorded at increasing temperatures and selected frequencies in the $0.1-1500 \mathrm{~Hz}$ range, after an initial cooling from RT down to $4.5 \mathrm{~K}$ in the absence of the field [zero field cooled (ZFC) procedure], on a SQUID magnetometer model MPMS2 at ICMA, Universidad de Zaragoza.

\section{RESULTS AND DISCUSSION}

The RT XRD pattern of the dU61Fh sample is presented in Fig. 1. The patterns of the d-U(2000) matrix and of a poly(ethylene glycol) (PEG) $\left[\mathrm{HO}-\left(\mathrm{CH}_{2}-\mathrm{CH}_{2}-\mathrm{O}\right)_{n}-\mathrm{H}\right.$, $\left.M_{w}=2000\right]$ sample are also shown. PEG, a low molecular weight poly(oxyethylene), may be considered as a close ana$\log$ of the short POE segments present in the organic spacer of dU61Fh. The dU6lFh diffraction pattern displays a set of peaks (the most intense at ca. $19.2^{\circ}$ and $23.4^{\circ}$ ) also present in the patterns of d-U(2000) matrix and in the PEG pattern. Therefore, these peaks are associated with crystalline POE 

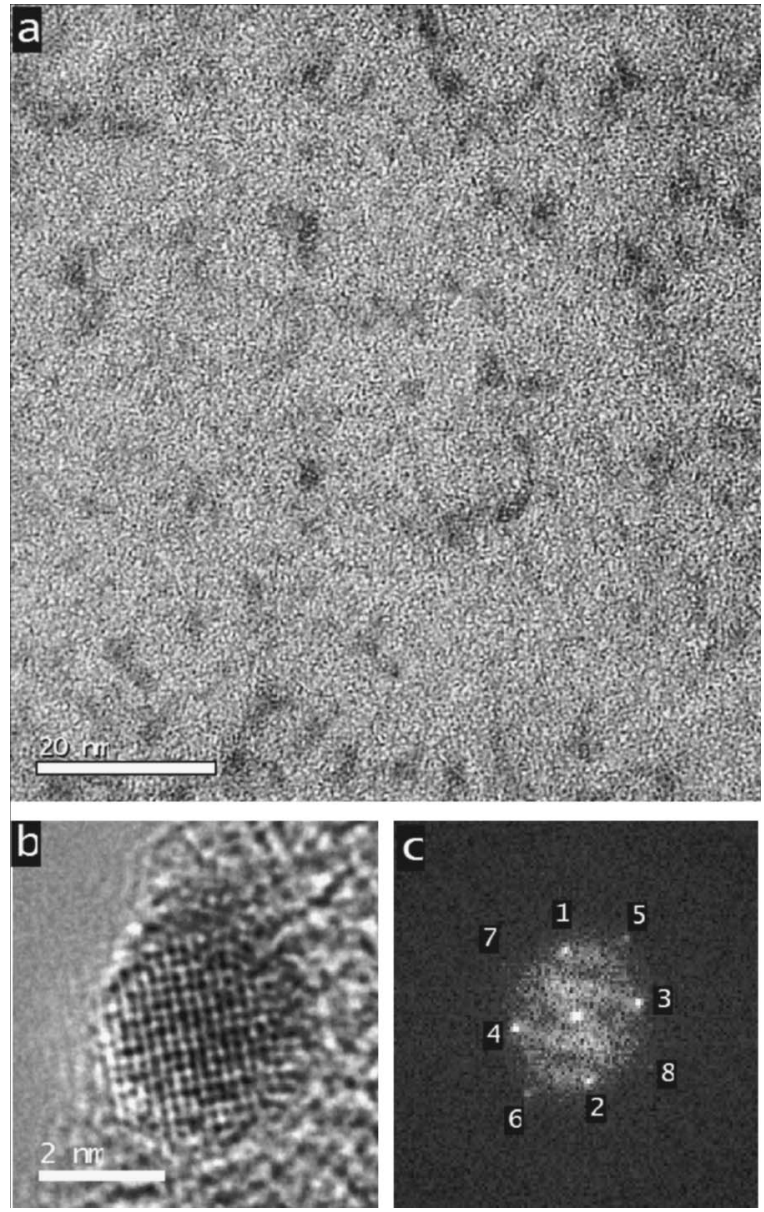

FIG. 2. (a) Bright field TEM image of ferrihydrite nanoparticles within the hybrid diureasil matrix. (b) High resolution image of a particle located at the edge of the sample. (c) Fourier transform of the area shown in (b). The obtained spots are labeled and the data are displayed in Table I.

regions. ${ }^{16}$ The dU61Fh diffraction pattern displays also a broad peak centered at ca. $21^{\circ}$ associated with ordering within the siloxane domains. ${ }^{16}$ The second order of this peak appears as a broad weak hump around $39^{\circ}-44^{\circ}$. The broad peak at ca. $62^{\circ}$ (signed with ${ }^{*}$ in Fig. 1, inset) indicates the existence of an iron-based phase. After a thermal treatment at $150{ }^{\circ} \mathrm{C}$ the POE chains become amorphous and broad peaks at ca. $35^{\circ}$ and $62^{\circ}$ are apparent. These peaks correspond to the most intense features of ferrihydrite. The peak at $35^{\circ}$ corresponds to a characteristic distance of $0.2-0.3 \mathrm{~nm}$ that can be associated with the 110 and 111 reflections $(0.250$ and $0.251 \mathrm{~nm}$, respectively), according to the model of Drits et al. ${ }^{6}$ However, more detailed studies are needed to clearly establish the origin of these peaks and determine the associated characteristic size, which cannot be estimated via the Debye-Scherrer law.

In Fig. 2(a), a TEM bright field image of the dU61Fh sample is shown, in which particles with various sizes within an amorphous matrix can be distinguished. Because of the small particle size and the presence of the matrix, the edges of the particles cannot be clearly discerned. However, we do get sufficient contrast to confirm the presence of nanosized particles. The average particle size obtained from measurements on different particles in Fig. 2(a) is $5 \mathrm{~nm}$.

Figure 3(a) corresponds to a different area from the same
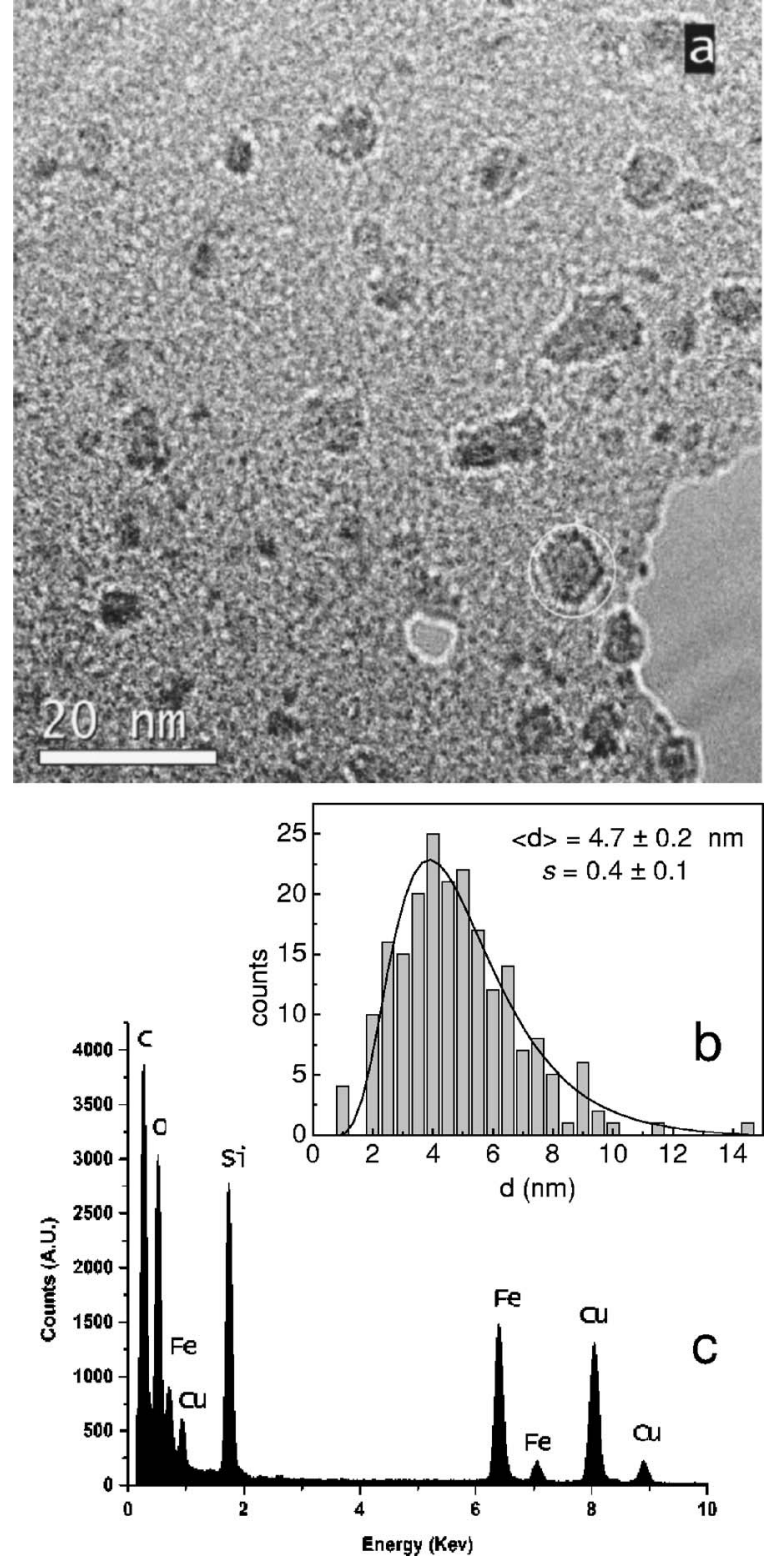

FIG. 3. (a) Bright field TEM image of ferrihydrite nanoparticles within the hybrid diureasil matrix. (b) Microanalysis measured in the area within the circle drawn in (a). (c) Particles size histogram.

sample in which we found particles ranging within $2-11 \mathrm{~nm}$, again with an average of $5 \mathrm{~nm}$ obtained from a larger number of particles. In this image, the particle edges are more clearly discerned, probably because the thickness of the film is smaller than that in Fig. 2(a). TEM images from various regions within the samples almost invariably show isolated nanoparticles and no signs of coalescence. The obtained particle sizes are of the same order of magnitude as those previously reported by Janney et al. ${ }^{8}$, who also showed particles with nonfacetted and rather fuzzy edges. A particle size histogram was obtained by analyzing 200 particles [Fig. $3(b)]$. The size distribution can be described by a lognormal function, with an average diameter of $4.7 \pm 0.2 \mathrm{~nm}$ and deviation $s=0.4 \pm 0.1$. Using the average diameter and some other parameters one can estimate the density of particles $N_{p}$ (number of particles per volume of sample) and the number of Fe ions per average diameter particle $N_{t}$ as follows. Con- 
TABLE I. $d_{h k l}$ values obtained after Fourier transform of Fig. 2(b) and typical $d_{h k l}$ values of 21 and 61 ferrihydrite.

\begin{tabular}{cccc}
\hline \hline Spot & $\begin{array}{c}\text { Figure 2(b) } \\
\left(d_{h k l} \mathrm{~nm}\right)\end{array}$ & $\begin{array}{c}61 \mathrm{Fh} \\
\left(d_{h k l} \mathrm{~nm}\right)\end{array}$ & $\begin{array}{c}2 \mathrm{Fh} \\
\left(d_{h k l} \mathrm{~nm}\right)\end{array}$ \\
\hline 1 & 0.20 & 0.20 & $\ldots$ \\
2 & 0.20 & 0.20 & $\ldots$ \\
3 & 0.19 & 0.20 & $\ldots$ \\
4 & 0.19 & 0.20 & $\ldots$ \\
5 & 0.14 & 0.15 & 0.15 \\
6 & 0.14 & 0.15 & 0.15 \\
7 & 0.14 & 0.15 & 0.15 \\
8 & 0.13 & 0.15 & 0.15 \\
\hline \hline
\end{tabular}

sidering the iron content $\left(2.1 \% w_{\mathrm{Fe}} / w_{\text {sample }}\right)$, the matrix and ferrihydrite densities [respectively, 1.2 and $3.96 \mathrm{~g} / \mathrm{cm}^{3}$ (Ref. 1)] and the mean particle volume, the density of particles $N_{p}$ is of the order of $(0.8 \pm 0.3) \times 10^{17}$ particles $/ \mathrm{cm}^{3}$. Thus, the mean interparticle distance is about $20-30 \mathrm{~nm}$, in agreement with the TEM images. According to the model of Drits et al., ${ }^{6,17}$ ferrihydrite is constituted by defective and defectivefree structures with unit cell volumes of $3.54 \times 10^{-2}$ and $7.08 \times 10^{-2} \mathrm{~nm}^{3}$, respectively. ${ }^{17}$ In this model the numbers of Fe ions per unit cell of the defective and defective-free structures are 0.96 and 1.56 , respectively, and the volume ratio of the structures is typically about $1: 1 .^{17}$ Therefore, the number of $\mathrm{Fe}$ ions in a spherical particle with an average diameter of $4.7 \mathrm{~nm}, N_{t}$, is about 1300. Particles with full defective and defective-free structures would have $N_{t} \sim 1200$ and 1500 ions, respectively. Considering other models for ferrihydrite structure, namely, the Eggleton and Fitzpatrick ${ }^{18}$ and the Towe and Bradley models, ${ }^{19}$ one obtains similar $N_{t}$ values. Thus, in further analysis we will consider $N_{t}=1300 \pm 200$ ions.

The high resolution image in Fig. 2(b) was measured from a particle located right on the edge of the sample, so that the sample is thin enough to acquire a lattice image of the particle. In this image, we can see that the particle is crystalline, with a diameter of ca. $4 \mathrm{~nm}$ and surrounded by the amorphous hybrid matrix. The corresponding Fourier transform is shown in Fig. 2(c), with up to eight high intensity spots, from which the corresponding interplane distances were calculated. The experimental values are displayed in Table I and compared to the closer $d_{h k l}$ from both ferrihydrites $61 \mathrm{Fh}$ and $21 \mathrm{Fh}$. From this comparison, this particle appears to be a ferrihydrite type 61Fh, since it presents a $d_{h k l}$ of $0.20 \mathrm{~nm}$, which is only possible in such a ferrihydrite. In fact, 61Fh usually appears after a synthesis procedure similar to that used here, i.e., thermal treatment of an iron nitrate solution at low $p \mathrm{H}$, while $2 \mathrm{lFh}$ is often the product of the basic treatment of an iron nitrate solution. ${ }^{7}$

For a complete analysis of the material we need to obtain an elemental distribution within the sample, as well as the composition of nanoparticles and matrix. This was done by means of EDS. In Fig. 3(c) we show the EDS spectrum measured from the area within the circle drawn in Fig. 3(a), comprising almost exclusively a single particle with a diameter of $10 \mathrm{~nm}$. The spectrum confirms the presence of iron in the particle, and we also see peaks from carbon, oxygen, and

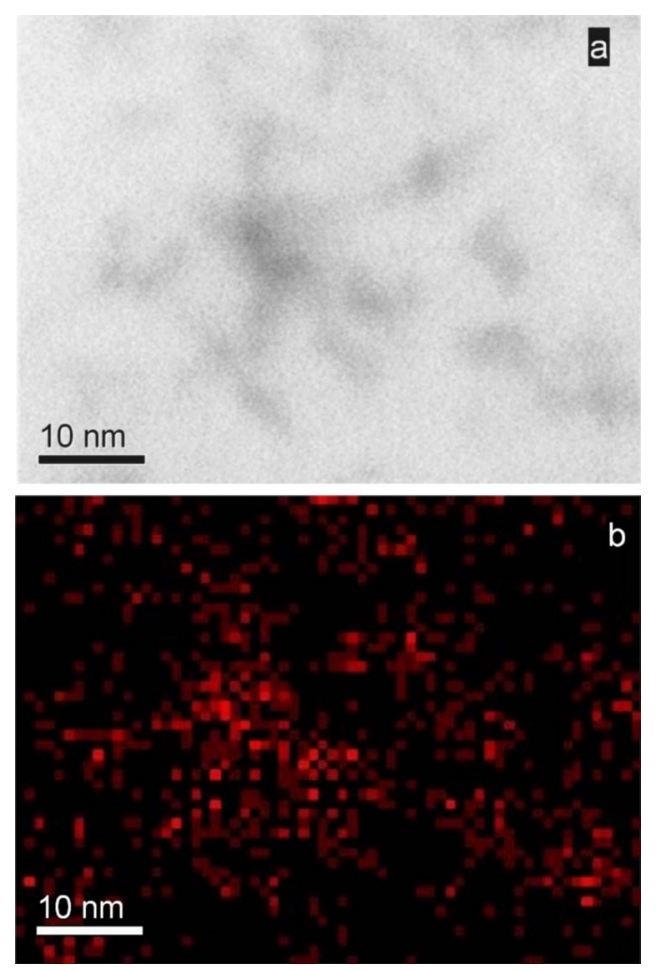

FIG. 4. (Color online) (a) STEM image obtained with a bright field detector. (b) Element distribution mapping of Fe obtained using the $K \alpha$ iron line.

silicon, which were expected for the matrix. As usual, copper peaks are also measured from the supporting grid. Since EDS provides information from the whole area, we decided to use a higher resolution analytical technique to confirm that iron is located in the particles, not in the matrix.

We used the compositional image technique through EDS in STEM. An example of the results is displayed in Fig. 4, including a STEM image of several particles generated with the bright field detector [Fig. 4(a)], and an elemental distribution mapping of $\mathrm{Fe}$, generated from the Fe $K \alpha$ emission line [Fig. 4(b)], for the same area of the STEM image. In the STEM image we can see in dark contrast the particle location within the matrix and with brighter contrast the matrix itself. In this image we see small particles as well as larger particles (which presumably arise from superposition of particles at various heights), ranging between 2 and $10 \mathrm{~nm}$. Again, the particles are not perfectly discerned because of the matrix around them. Since the matrix around the particles also contains oxygen, the only element we can use to distinguish the ferrihydrite from the matrix is iron. As shown in Fig. 4, the detection maxima within the mapping of Fe coincide with the darker areas within the STEM image, which are expected to correspond to ferrihydrite particles. The quality of the mapping is hindered by the exposure time, which is in turn limited by the image drift induced by the electron beam, but even with this limitation we see a good agreement. It is important to stress that the Fe signal is only observed in the areas with particles, so that we can discard the presence of iron in the matrix around them.

The in-phase component of the magnetic susceptibility of the dU61Fh sample, $\chi^{\prime}$, is plotted in Fig. 5 as a function of temperature, for selected frequencies in the $0.1-1500 \mathrm{~Hz}$ 


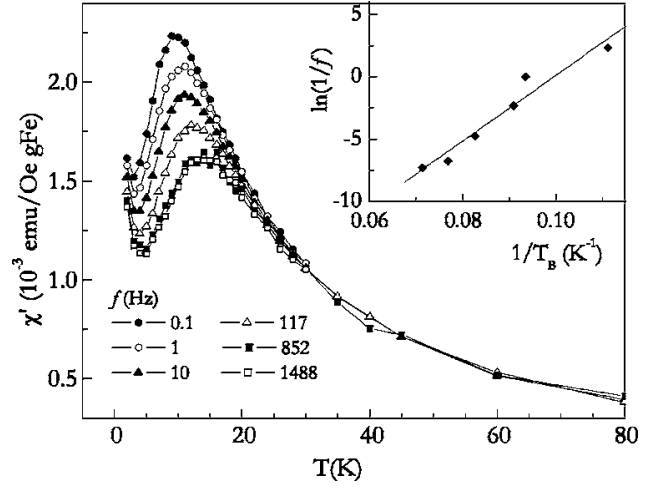

FIG. 5. In-phase ac susceptibility of the diureasil/ferrihydrite hybrid sample as a function of temperature. Inset: linearization of the Néel-Arrhenius relation, relating the measured frequency $f$ and the blocking temperature $T_{B}$.

range. Above $T_{F}=30 \mathrm{~K}, \chi^{\prime}$ is independent of the applied field frequency and follows approximately a Curie law. That is, magnetic moments are able to cross the anisotropy energy barrier in the time scale of the experiments. For decreasing temperatures and below $30 \mathrm{~K}$, the particles that experience higher energy barriers become blocked. This leads to a decrease of $\chi^{\prime}$ compared to that expected from the Curie law and to the onset of the out-of-phase susceptibility component, $\chi^{\prime \prime}$. At a temperature $T_{B}$, resulting from the balance between the decrease resulting from the blocking and the increase predicted by Curie law, the in-phase susceptibility curves of the dU61Fh sample (Fig. 5) exhibit a frequencydependent maximum. This dependence follows a NéelArrhenius relation: ${ }^{20} f=f_{0} \exp \left(-E / k_{B} T\right)$, expected for superparamagnetic particles (Fig. 5, inset). $f_{0}$ is a microscopic attempt frequency, $E$ is the anisotropy energy barrier to reversal of magnetization, and $k_{B}$ is the Boltzmann constant. From the $T_{B}(f)$ dependence, $E$ and $\tau_{0}=1 / f_{0}$ are estimated to be $260 \pm 30 \mathrm{~K}$ and $1 \times 10^{-12} \mathrm{~s}$, respectively. From the anisotropy energy barrier $E$, one can determine the effective anisotropy constant $K_{\text {eff }}$, since $E=K_{\text {eff }} V$, where $V$ is the particle volume. Considering spherical particles and the average size obtained form TEM measurements $K_{\text {eff }}=4 \times 10^{5} \mathrm{erg} / \mathrm{cm}^{3}$, in agreement with the value found in artificially reconstructed ferritin cores $\left[(3-6) \times 10^{5} \mathrm{erg} / \mathrm{cm}^{3}\right]{ }^{21}$ We remark that an extrapolated $\tau_{0}$ of the order of $10^{-10}-10^{-12}$, as found in the dU61Fh sample, is characteristic of noninteracting/very weakly interacting particles. On the contrary, interacting nanoparticles have much lower extrapolated $\tau_{0}$ values. ${ }^{22}$

In the nonblocked superparamagnetic regime, i.e., for $T>30 \mathrm{~K}$, the magnetization curves of the dU61Fh sample show a component which is linear with the field superimposed to the saturation and no hysteresis [Fig. 6(a)]. The linear component was separated from the saturation one using a scale method. ${ }^{23}$ The latter was fitted to a Langevin distributed function,

$$
M_{S}(H, T)=N_{p} \int_{\mu_{\min }}^{\mu_{\max }} \mu L\left(\frac{\mu H}{k T}\right) f(\mu) d \mu,
$$

where $N_{p}$ is the density of particles, $\mu$ is the particle moment, and $f(\mu)$ its normalized lognormal distribution. The fit yielded $\langle\mu\rangle=60 \mu_{B}$ [at $45 \mathrm{~K}$, shown in Fig. 6(b)], decreasing

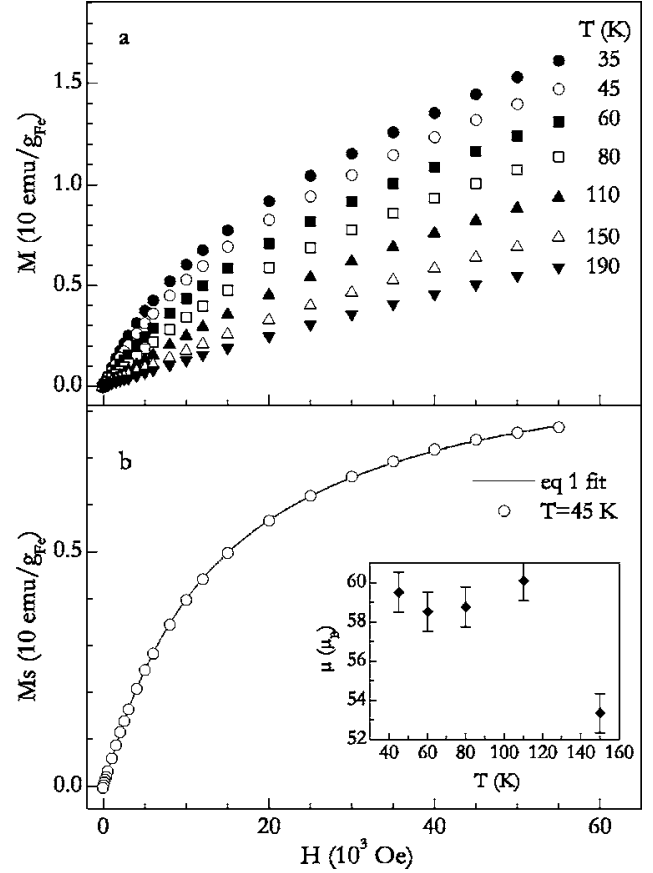

FIG. 6. (a) Magnetization curves $M(H)$ at selected temperatures. (b) Saturation component of the magnetization at $45 \mathrm{~K}$ and Langevin lognormal distributed [Eq. (1)] fit. Inset of (b) shows the variation of the fitted average moment $\langle\mu\rangle$ with temperature.

to about $80 \%$ of this value at $T=150 \mathrm{~K}$ [Fig. 6(b), inset], $s$ $=0.9$, and $N_{p}=2 \times 10^{17}$ particles $/ \mathrm{cm}^{3} . N_{p}$ and $s$ are constants, within the fit error and in the studied temperature range. The $N_{p}$ value derived here agrees qualitatively well with the value obtained from TEM images. The mean magnetic moment corresponds to a mean number of fully uncompensated $\mathrm{Fe}^{3+}$ ions $N_{\text {un }}$ of 12. Taking into account the value of $N_{t}$ derived above, $N_{\text {un }}=N_{t}^{p}$, with $p \approx 1 / 3$, which suggests that the uncompensated spins are mainly randomly distributed at the surface. In ferritin, the uncompensated spins are thought to be also randomly distributed through the volume, since $p$ approaches $1 / 2 .^{21,23}$

Finally, one can estimate the surface shell thickness corresponding to 12 uncompensated ions random distributed at the surface. Since they are randomly distributed, the number of ions among which 12 uncompensated ions can be found is of the order of $12^{2}$. Therefore, the determination of the surface shell thickness comprises finding the volume occupied by $N_{\text {un }}^{2}$ in the outer shell of a $4.7 \mathrm{~nm}$ diameter particle. The shell thickness is about $0.1-0.2 \mathrm{~nm}$, i.e., of the order of one ferrihydrite unit cell. This value is of the order of the one estimated for maghemite nanoparticles [between 0.35 and $1 \mathrm{~nm}$ (Refs. 24 and 25)].

\section{CONCLUSIONS}

Detailed TEM and STEM/EDS studies show the existence of dispersed six-line ferrihydrite nanoparticles in irondoped organic-inorganic composites. The mean particle size and size distribution were determined by TEM and compared to magnetic measurements. This allowed the determination of the anisotropy constant and the power relation, $p$, between number of iron ions per particle and the number of uncom- 
pensated ones. Since $p \approx 1 / 3$, the uncompensated spins are mainly random distributed at the surface. According to this model, a shell thickness of about one ferrihydrite unit cell is estimated.

\section{ACKNOWLEDGMENTS}

The authors acknowledge M. R. Soares for performing the DRX measurements and IFIMUP for the possibility of using SQUID. The financial support from FCT, POCTI/ CTM/46780/02, research grant MAT2004-03395-C02-01 from the Spanish CICYT, and Acción Integrada LusoEspañola E-105/04 is gratefully recognized. One of the authors (N.J.O.S.) acknowledges a grant from FCT (Grant No. SFRH/BD/10383/2002). Another author (L.M.L.-M.) acknowledges support from Xunta de Galicia (Grant No. PGIDIT03TMT30101PR).

${ }^{1}$ J. L. Jambor and J. E. Dutrizac, Chem. Rev. (Washington, D.C.) 98, 2549 (1998).

${ }^{2}$ S. Mann, in Inorganic Materials, 2nd ed., edited by S. Mann, J. Webb, R. J. P. Williams (Wiley, Chichester, 1996)

${ }^{3}$ S. Gider, D. D. Awschalom, T. Douglas, S. Mann, and M. Chaparala, Science 268, 77 (1995).

${ }^{4}$ L. Néel, Acad. Sci., Paris, C. R. 252, 4075 (1961).

${ }^{5}$ M. Fleischer, G. Y. Chao, and A. Kato, Am. Mineral. 60, 485 (1975).

${ }^{6}$ V. A. Drits, B. A. Sarkharov, A. L. Salyn, and A. Manceau, Clay Miner. 28, 185 (1993).

${ }^{7}$ U. Schwertmann, J. Friedl, and Helge Stanjek, J. Colloid Interface Sci. 209, 215 (1999).
${ }^{8}$ D. E. Janney, J. M. Cowley, and P. R. Buseck, Am. Mineral. 86, 327 (2001).

${ }^{9}$ T. Douglas and M. Young, Nature (London) 393, 152 (1998).

${ }^{10}$ J. A. Cowen, K. L. Tsai, and J. L. Dye, J. Appl. Phys. 76, 6567 (1994).

${ }^{11}$ R. F. Ziolo, E. P. Giannelis, B. A. Weinstein, M. P. O’Horo, B. N. Ganguly, V. Mehrotra, M. W. Russell, and D. R. Huffman, J. Appl. Phys. 257, 219 (1992)

${ }^{12}$ N. J. O. Silva, V. S. Amaral, V. de Zea Bermudez, S. C. Nunes, D. Ostrovskii, J. Rocha, and L. D. Carlos, J. Mater. Chem. 15, 484 (2005).

${ }^{13}$ N. J. O. Silva, K. Dahmouche, C. V. Santilli, V. S. Amaral, L. D. Carlos, V. de Zea Bermudez, and A. F. Craievich, J. Appl. Crystallogr. 36, 961 (2003).

${ }^{14}$ N. J. O. Silva, V. S. Amaral, L. D. Carlos, and V. de Zea Bermudez, J. Appl. Phys. 93, 6978 (2003).

${ }^{15}$ V. de Zea Bermudez, L. C. Carlos, and L. Alcácer, Chem. Mater. 13, 569 (1999).

${ }^{16}$ L. D. Carlos, V. de Zea Bermudez, R. A. Sá Ferreira, L. Marques, and M. Assunção, Chem. Mater. 11, 581 (1999).

${ }^{17}$ E. Jansen, A. Kyek, W. Schafer, and U. Schwertmann, Appl. Phys. A: Mater. Sci. Process. 74, S1004 (2002).

${ }^{18}$ R. A. Eggleton and R. W. Fitzpatrick, Clays Clay Miner. 36, 111 (1988) ${ }^{19}$ K. M. Towe and W. F. Bradley, J. Colloid Interface Sci. 24, 384 (1967)

${ }^{20}$ L. Néel, Acad. Sci., Paris, C. R. 228, 664 (1949); W. F. Brown, Jr., Phys. Rev. 130, 1677 (1963).

${ }^{21}$ C. Gilles, P. Bonville, K. K. W. Wong, and S. Mann, Eur. Phys. J. B 17, 417 (2000).

${ }^{22}$ J. L. Dormann, F. D’Orazio, F. Lucari, E. Tronc, P. Prené, J. P. Jolivel, D. Fiorani, R. Cherkaoui, and M. Noguès, Phys. Rev. B 53, 14291 (1996).

${ }^{23}$ N. J. O. Silva, V. S. Amaral, and L. D. Carlos, Phys. Rev. B 71, 184408 (2005).

${ }^{24}$ J. M. D. Coey, Phys. Rev. Lett. 27, 1140 (1971).

${ }^{25}$ S. Linderoth, P. V. Hendriksen, F. Bodker, S. Wells, K. Davies, and S. W. Charles, J. Appl. Phys. 75, 6583 (2004). 